\section{IMMUNE PROFILING OF PATIENTS WITH ADVANCED SOLID TUMORS TREATED WITH INTRATUMORALLY ADMINISTERED CV8102 AS A SINGLE-AGENT OR IN COMBINATION WITH ANTI-PD-1 ANTIBODIES IN PHASE I CLINICAL TRIAL}

\begin{abstract}
${ }^{1}$ Paula Codó*, ${ }^{2}$ Thomas Eigentler, ${ }^{3}$ Lucie Heinzerling, ${ }^{4}$ Juergen Krauss, ${ }^{5}$ Carsten Weishaupt, ${ }^{6}$ Sebastian Ochsenreither, ${ }^{7}$ Celeste Lebbe, ${ }^{8}$ Peter Mohr, ${ }^{9}$ Marc Oliva, ${ }^{10}$ Honey Oberoi, ${ }^{11}$ Patrick Terheyden, ${ }^{12}$ José Trigo Pérez, ${ }^{13}$ Franz-Georg Bauernfeind, ${ }^{14}$ Michael Fluck, ${ }^{15}$ Erika Richtig, ${ }^{16}$ Ainara Soria, 'Marina Gonzalez, ${ }^{1}$ Fatma Funkner, ${ }^{1}$ Peter Wengenmayer, 'Dominik Vahrenhorst, ${ }^{1}$ Tobias Seibel, ${ }^{1}$ Gianluca Quintini, ${ }^{1}$ Beate Schmitt-Bormann, ${ }^{1}$ Birgit Scheel, ${ }^{1}$ Martin Falk, 'Ulrike Gnad-Vogt. 'CureVac AG, Frankfurt, Germany; ${ }^{2}$ University Medical Center Tübingen, Tübingen, Germany; ${ }^{3}$ University of Erlangen, Erlangen, Germany; ${ }^{4}$ National Center for Tumor diseases (NCT), Heidelberg, Germany; ${ }^{5}$ University of Münster, Münster, Germany; ${ }^{6}$ Charité Comprehensive Cancer Center, Berlin, Germany; ${ }^{7}$ Hôpital Saint Louis, Paris, France; ${ }^{8}$ Elbe Medical Center, Buxtehude, Germany; ${ }^{9}$ Institut Catala d'Oncologia (ICO), Barcelona, Spain; ${ }^{10}$ Vall d'Hebron University Hospital, Barcelona, Spain; ${ }^{11}$ University of Lübeck, Lübeck, Germany; ${ }^{12}$ Hospital Clínico V de la Victoria, Málaga, Spain; ${ }^{13}$ University Clinic Bonn, Bonn, Germany; ${ }^{14}$ Fachklinik Hornheide, Münster, Germany; ${ }^{15}$ Medical University of Graz, Graz, Austria; ${ }^{16}$ Hospital Ramon y Cajal, Madrid, Spain
\end{abstract}

Background CV8102 is a non-coding, non-capped RNA complexed with a carrier peptide activating the innate (via TLR7/ 8 , RIG-I) and adaptive immune system. ${ }^{1}{ }^{2}$ An ongoing phase I trial is investigating the intratumoral (i.t.) administration of CV8102 in patients with advanced cutaneous melanoma (cMEL), squamous cell carcinoma of the skin (cSCC) or head and neck (hnSCC) and adenoid cystic carcinoma (ACC), either as a single agent or in combination with systemic anti-PD-1 antibodies. Preliminary immune profiling results will be reported.

Methods An open-label, cohort-based, dose escalation and expansion study in patients with advanced cMEL, cSCC, hnSCC or ACC is ongoing investigating CV8102 i.t. as single agent and in combination with anti-PD-1 antibodies. Eight i.t. injections of CV8102 were administered over a 12 week period with optional continuing treatment in case of clinical benefit. In the initial dose escalation part, the recommended phase II dose for subsequent cohort expansion was defined. Blood samples for immune cell phenotyping, RNA sequencing (RNAseq) and serum cytokine/chemokine analysis were collected at baseline and multiple time points during the treatment period. For characterization of the tumor microenvironment (TME), optional core needle biopsies of injected and/or non-injected lesions were taken before, during and after treatment. Changes on various tumor-infiltrating immune cells were assessed by multiplex immunofluorescence (MultiOmyx $<$ sup $>$ TM $<$ /sup $>$ ) and immune-related gene expression profiling using nCounter ${ }^{\circledR}$ Pan Cancer IO360 < sup $>\mathrm{TM}</$ sup $>$ panel (NanoString).

Results During the dose escalation part, 33 patients received CV8102 (dose range of $25-900 \mu \mathrm{g}$ ) as single agent and 25 patients received CV8102 in combination with an anti-PD-1 antibody. A dose of $600 \mu \mathrm{g}$ was selected as recommended phase II dose. Serum cytokine/chemokine and blood RNAseq analysis showed transient increases in several markers like interferons alpha and gamma after the first dose. First analyses of paired biopsies showed changes in the TME of injected and non-injected lesions. Complete results of cytokine and chemokine analysis in serum and blood RNAseq for the dose escalation cohorts will be presented. Multiplex immunofluorescence and gene expression profiling from paired biopsies from individual patients will be also included.

Conclusions Intratumoral injection of CV8102 activated several cytokine/chemokine pathways in the peripheral blood and showed immunological changes in the tumor microenvironment of injected and non-injected lesions.

Trial Registration NCT03291002

\section{REFERENCES}

1. Ziegler $A$, Soldner $C$, Lienenklaus $S$, Spanier J, Trittel $S$, Riese $P$, Kramps $T$, Weiss $S$, Heidenreich R, Jasny E, Guzmán CA, Kallen KJ, Fotin-Mleczek M, Kalinke U. A New RNA-Based Adjuvant Enhances Virus-Specific Vaccine Responses by Locally Triggering TLR- and RLH-Dependent Effects. J Immunol 2017;198(4):1595-1605. doi: 10.4049/jimmunol.1601129.

2. Heidenreich R, Jasny E, Kowalczyk A, Lutz J, Probst J, Baumhof P, Scheel B, Voss $S$, Kallen KJ, Fotin-Mleczek M. A novel RNA-based adjuvant combines strong immunostimulatory capacities with a favorable safety profile. Int J Cancer 2015 Jul 15;137(2):372-84. doi: 10.1002/ijc.29402.

Ethics Approval The study was approved by the Central Ethics Committees in Tuebingen, Germany under 785/2016AMG1, in France by the COMITE DE PROTECTION DES PERSONNES SUD-EST I under 2019-49, approval dated 17-May2019, in Barcelona, Spain by the CEC COMITÉ DE ÉTICA DE INVESTIGACIÓN CLÍNICA CON MEDICAMENTOS del Hospital Universitari Vall d'Hebron, approval date 28-Nov2019 under the EUdraCT number, in Austria by the Central Ethics Committee in Graz under 31-426 ex 18/19 approved on 19-Sep-2019.

Consent Written informed consent from the patient was obtained for publication of this abstract and any accompanying images. A copy of the written consent is available for review by the Editor of this journal.

http://dx.doi.org/10.1136/jitc-2021-SITC2021.473 